\title{
THE PROGNOSTIC VALUE OF CHOLESTERINEMIA IN CHRONIC NEPHRITIS
}

FINAL, REPORT

\author{
EDWIN HENES, JR., A.B., M.D. \\ MIJWAUKEE
}

In a preliminary report on this subject, ${ }^{1}$ I presented a number of cases to show that. a hypercholesterinemia is usually to be found in cases of chronic nephritis which are progressing favorably, and that a normal or subnormal figure for the serum cholesterol, in cases of chronic nephritis, is of distinct prognostic importance and value. The measure of cholesterinemia in the cases enumerated in my early paper accurately foretold the prognosis in individual cases.

The study of the chemistry of the blood has made giant strides during the past few years, and we are now enabled to measure accurately, especially the nonnitrogenous constituents of the blood - total nonprotein nitrogen, urea nitrogen, creatinin, uric acid and sugar. There seems to be little disagreement as to the best quantitative methods to employ, and the clinician has a right to accept the quantitative determination without a doubt.

Much work along this line has been done, but notably in nephritis and diabetes mellitus, conditions in which the carbon dioxid combining power of the blood is of exceedingly great value from a prognostic standpoint.

While there is no doubt that the study of the prognosis in chronic nephritis must, of necessity, be associated closely with those chemical metabolic products which the kidneys normally excrete, and which, in the event of functional decompensation, are excreted with increasing difficulty, resulting in a corresponding increase of these same substances in the circulating blood, there is evidence to show that the uremic death of nephritics is due to a severe acidosis. The marked fall in the carbon dioxid combining power of the blood in uremia is a striking proof of this, and it has been my belief for many years that the lipoid substances of the blood are intimately associated in the chemistry of acidosis.

With the advent of so-called "blood chemistry," investigators actually plunged into the study of chronic nephritis with exceedingly valuable results. I have always insisted, however, that the study of cholesterinemia in chronic nephritis was equally as important, if not more

1. New York State M. J. 15:300 (Aug.) 1915. 
so, in an endeavor to prognosticate in any given case. The work begun in 1914 was, therefore, continued. It is my purpose to show how intimately associated are the study of the cholesterinemia and the study of the nonnitrogenous products of the blood in chronic nephritis, and at the same time, to add further weight in substantiation of my preliminary report on the subject.

Reference to the preliminary report will show that the measure of cholesterinemia in the individual cases presented foretold the prognosis very accurately in each case. It is to be remembered that the cholesterinemia must be interpreted properly in each case, and allowances must be made for any coincident clinical conditions that experience has taught us tend to increase or decrease the amount of cholesterol in the blood. The early report showed that a hypercholesterinemia is present in all cases of chronic nephritis, and that the measure of cholesterinemia decreases as the uremic state is approached. All cases of uremia, and all cases terminating fatally in coma, showed a nost decided hypocholesterinemia.

Since the introduction of blood chemistry, the functional ability of the kidneys has been studied in terms of the retention in the blood of the total nonprotein nitrogen, urea, creatinin and uric acid, and the carbon dioxid combining power of the blood serum. Our studies have shown that evidence of increasing retention in the blood of the products just enumerated is of prognostic importance, and rather accurately foretells an impending uremic state.

The blood figures generally recognized as being the normal for the blood constituents entumerated, are as follows:

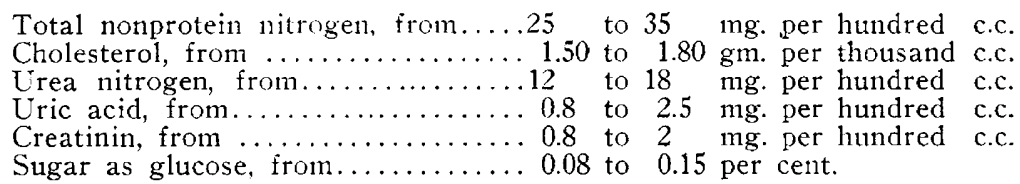

Any increase in the figures mentioned is to be regarded as evidence of retention of these products, but in speaking of cholesterol, it is probably not correct to speak of it as being retained, for, in the normal individual it is not a product of excretion. When figures as high as $80 \mathrm{mg}$. of nonprotein nitrogen per hundred c.c. of blood are obtained, a very definite evidence of retention of these waste nitrogenous products is indicated. When figures as low as $1 \mathrm{gm}$. of cholesterol per thousand of blood are obtained, they are distinctly below normal, especially in a condition where a decided increase is to be expected. It is just this normal, or subnormal figure for the cholesterol in chronic nephritis, which is of such interest and importance. 
REPORT OF CASES

The following cases will show the prognostic import that can be attached to the measure of cholesterinemia in chronic nephritis.

CASE 1.--Male, aged 66; no jaundice; no fever; moderate arteriosclerosis; blood pressure, 220; Wassermann reaction, negative; urine contained albumin, hyaline and granular casts. The general condition of the patient was not very good at the time the blood was examined. Examination of the blood showed: urea nitrogen, $55 \mathrm{mg}$; uric acid, $2.7 \mathrm{mg}$; creatinin, $1 \mathrm{mg}$, and sugar 0.132 per cent., while the cholesterol was $2.05 \mathrm{mg}$.

Under the clinical circumstances one would expect a much higher cholesterol figure. The absence of a decided hypercholesterinemia in this case should make me suspect a moderate degree of retention, and the figures for the nonnitrogenous products indicate that retention.

In presenting these cases, only relevant clinical states, as they influence cholesterinemia, are recorded. These clinical states are the following: (1) Fever reduces cholesterinemia; (2) jaundice increases cholesterinemia; (3) arteriosclerosis (active) increases cholesterinemia.

CASE 2.-Female, aged 60 ; temperature, $100.8 \mathrm{~F}$., no jaundice; slight arteriosclerosis; urine contained albumin. hyaline and granular casts; no edema; eye grounds negative; Wassermann negative. At the time the blood was examined, there were no symptoms suggesting an impending uremic state, but the cholesterinemia amounted to only $1.73 \mathrm{mg}$., a hypocholesterinemia under the circumstances. On the following day, a sudden delirium set in, the patient gradually went into coma and died two days later.

In this case, while there are no additional blood figures, I am reasonably sure that examination would have shown evidence of a decided retention of waste nitrogenous products.

CASE 3.-Female, aged 59; was admitted to the hospital with a diagnosis of carcinoma of the stomach, although repeated roentgen-ray examinations failed to substantiate that diagnosis. The temperature was $98 \mathrm{~F}$.; the Wassermann reaction was negative; hemoglobin, 52 per cent.; red blood corpuscles, 2,420,000; leukocytes, 9,200; polymorphonuclears, 66 per cent.; no jaundice; urine showed evidences of nephritis.

Our attention was drawn to her when she began to vomit and became drowsy, although previous to this, the patient manifested no signs of an impending uremia. The blood figures were as follows: Cholesterol, $1.65 \mathrm{mg}$; nonprotein nitrogen, $1.25 \mathrm{mg}$; urea nitrogen, $100 \mathrm{mg}$; uric acid, $5.5 \mathrm{mg}$; creatinin, $13.4 \mathrm{mg}$; sugar, 0.129 per cent.; carbon dioxid, 10 volumes per hundred c.c.

These figures certainly suggest a threatened uremia. The stupor continued, coma supervened and the patient died at the end of two days.

CASE 4.-Male, aged 50; admitted to the hospital with a diagnosis of chronic nephritis; no jaundice; temperature, $99 \mathrm{~F}$.; Wassermann negative; blood pressure, 180, moderate arteriosclerosis; urine contained albumin and hyaline and granular casts; no edema; general condition good. The following figures were obtained: Phenolphthalein elimination, 79 per cent.; urea nitrogen, $17.5 \mathrm{mg}$.; uric acid, $1.4 \mathrm{mg}$; ; creatinin, $1.5 \mathrm{mg}$.; blood sugar, 0.09 per cent., and cholesterol $2.34 \mathrm{mg}$. 
Having done well, the patient was discharged, but returned to the hospital a year later. On his second admission, he was restless, noisy, not mentally clear, had a motor aphasia, was drowsy and sleepy, but showed no convulsions. His blood pressure was $170 / 110$; no jaundice; no fever; urine contained albumin and hyaline and granular casts.

We were here dealing with an undoubted case of threatened uremia, and the blood figures obtained verified the condition. They were: nonprotein nitrogen, 110; urea nitrogen, 58; creatinin, 3.5 ; uric acid, $5 \mathrm{mg}$. and sugar, 0.136 per cent., with a carbon dioxid combining power of 42 volumes per hundred c.c. The cholesterinemia amounted to $1.43 \mathrm{mg}$., a decidedly low figure, under the circumstances.

Two weeks later the general condition of this patient was somewhat improved, but at the end of another week, the general condition again became worse and the following blood figures were obtained: nonprotein nitrogen, 41 mg.; urea nitrogen, $16 \mathrm{mg}$; uric acid, $1 \mathrm{mg}$.; creatinin, $1.1 \mathrm{mg}$; sugar, 0.146 per cent.; carbon dioxid, 48.5 volumes per hundred c.c. These are practically normal figures, but the cholesterinemia continued low, $2.02 \mathrm{mg}$. Subsequent work was not done in this case, but the patient, after some ups and downs, eventually died in uremic coma.

CASE 5.-Female, aged 61 ; was admitted to the hospital with a mild chronic nephritis and arteriosclerosis; no jaundice; no fever; blood pressure, 210; Wassermann, negative; urine, very faint trace of albumin and no casts. Eyegrounds showed neurochorioretinitis. A facial palsy, with a pure motor aphasia, justified the diagnosis of hemorrhage from the middle meningeal or middle cerebral artery.

The blood figures in this case were as follows: Urea nitrogen, $16 \mathrm{mg}$; uric acid, $2.7 \mathrm{mg}$.; creatinin, $1.5 \mathrm{mg}$; sugar, 0.106 per cent., and cholesterol, $2.93 \mathrm{mg}$. These figures certainly do not suggest a severe chronic nephritis. Four days later, a sudden coma developed and the patient died. Death was most likely due to cerebral hemorrhage.

CASE 6.-Male, aged 52; a case of chronic nephritis with undoubted uremic state, for when the blood was first examined the patient was in coma. Quantitative estimations of the nonprotein nitrogenous constituents of the blood at that time gave the following: Urea nitrogen, $35 \mathrm{mg}$; creatinin, $1.3 \mathrm{mg}$; sugar, 0.112 per cent., and carbon dioxid combining power, 31 volumes per hundred c.c., figures that surely do not indicate a very severe retention. There was no jaundice; blood pressure was 150 ; no edema and the urine showed albumin and hyaline and granular casts. The cholesterinemia amounted to $1.77 \mathrm{mg}$. a decidedly low figure under the circumstances. With sodium bicarbonate infusions the patient held his own for a few days. but a second examination of the blood failed to show the apparent gravity of the condition. The figures obtained were as follows: Urea nitrogen, 31 ; uric acid, 2.3 ; creatinin, 2.5 ; sugar, 0.12 per cent.; carbon dioxid combining power, 48 volumes. Ten days later the patient died in coma. In this case, the relative hypocholesterinemia was of correct prognostic importance, while the measures of the nonnitrogenous constituents were not.

CASE 7.-Male; aged 55; no jaundice; no fever; blood pressure, 170; moderate arteriosclerosis; Wassermann, negative; urine contained heavy trace of albumin, with hyaline and granular casts; general anasarca. Patient was restless, noisy and drowsy and a uremic state was certainly impending. Blood examination showed: nonprotein nitrogen, 144; urea nitrogen, 85 ; creatinin, 6 ; uric acid, 10; sugar, 0.152 per cent. and cholesterol, $1.85 \mathrm{mg}$. These figures bear out the severity of the condition. This patient grew steadily worse; delirium set in, and in two days he died in coma.

CASE 8.-Male; aged 67. This patient was uremic and in very poor condition. The urine contained albumin, but no casts, and no acetone bodies. The blood pressure was 185. A pulmonary congestion was responsible for a fever 
of $101.5 \mathrm{~F}$. and a leukocyte count of 16,500 . The figures for the blood were as follows: nonprotein nitrogen, 250; urea nitrogen, 150; uric acid, 10; creatinin, 18.7; sugar, 0.2 per cent. and carbon dioxid combining power 15; cholesterol, $0.65 \mathrm{mg}$. At the end of twenty-four hours, muscular twitchings developed, the patient became stuporous, went into coma, and died. Figures before death (and without fever) were as follows: nonprotein nitrogen, 250; urea nitrogen, 166; uric acid, 10; creatinin, 18; sugar, 0.24 per cent.; carbon dioxid combining power 44 , and cholesterol $0.97 \mathrm{mg}$. These figures are typical of a uremic coma. At no time did this patient eliminate any phenolsulphonephthalein.

CASE 9.-Male; aged 50; no fever; no jaundice; no arteriosclerosis; blood pressure, 200; Wassermann, negative; urine showed a large amount of albumin and hyaline and granular casts; dyspnea and general evidences of impending uremia, although the general condition of the patient was fairly good. Reasonably high figures for the waste products were found in examining the blood: nonprotein nitrogen, 55 ; urea nitrogen, 25 ; creatinin, 3.5 ; uric acid, 5 ; sugar, 0.2 per cent.; phenolsulphonephthalein elimination 38 per cent. The high figure for the uric acid is certainly suspicious. A decided relative hypocholesterinemia, $1.45 \mathrm{mg}$. was found. Ten days later, the patient became drowsy and stuporous and irrational and had to be tied in bed. Blood figures at this time, except for the cholesterol, again failed to show the gravity of the situation: urea nitrogen, 19 ; uric acid, 3.6 ; creatinin, 1.9 ; sugar, 0.10 per cent., while the cholesterol amounted to $1 \mathrm{mg}$., a very low figure under the circumstances. Two days later the patient went into coma and died.

CASE 10.-Male; aged 27. This was a case of nephritis, based on syphilis, and despite the condition of the kidneys, was given vigorous antisyphilitic treatment. This patient did very well while under observation, and at no time showed any uremic tendencies. This case is reported to show the blood figures that are to be expected. There was no jaundice; no fever; slight general edema; the urine contained.albumin and hyaline and granular casts; no headache; no vomiting; eyegrounds normal. Chemical examinations resulted as follows: Phenolsulphonephthalein elimination, 55; urea nitrogen, 15; creatinin, 1.5; sugar, 0.062 per cent., and cholesterol, $4.17 \mathrm{mg}$. Several months later the patient was under treatment in the dispensary, and was doing well. The cholesterinemia amounted to $3.49 \mathrm{mg}$. at that time. This patient was eventually lost sight of; however, continued observation should include a periodic examination of the blood cholesterol, and if it should be found to be steadily decreasing, the prognosis would be correspondingly worse. This fact is demonstrated by the following cases.

CASE 11.-Male, aged 35 ; no fever; no jaundice; no arteriosclerosis; blood pressure, 240; urine contained albumin and hyaline and granular casts; intense headache, suggesting brain tumor; nausea; vomiting and diminished vision (fundi showed albuminuric retinitis); no edema; no rigidity of the neck; phenolsulphonephthalein elimination, 40; urea nitrogen, 19; uric acid, 2.6; creatinin, 1.5; carbon dioxid combining power, 55 ; cholesterol, $3 \mathrm{mg}$.

Two weeks later, with little change in his general condition (with roentgenray examinations of skull and spinal puncture negative) and the blood picture remaining the same, the cholesterinemia was found to have fallen to $2.42 \mathrm{mg}$. At the end of another two weeks, the cholesterinemia had dropped to $1.80 \mathrm{mg}$. The general condition of the patient became steadily worse; another two weeks found him stuporous and drowsy and passing only from 300 to 500 c.c. of urine a day. At this time, with the temperature subnormal (95 F.) and the picture of the urine unchanged, the cholesterinemia was found to be $1.10 \mathrm{mg}$. The following day, muscular twitchings set in, coma supervened and the patient died.

Necropsy revealed kidneys of a typical chronic interstitial nephritis. This case shows very well that it is the fall in the cholesterinemia that is of such prognostic importance. 
The final case to be presented is one of exceedingly great interest. It is a case in which repeated examinations of the blood had been made, a case that had been carefully studied. The blood figures show well how the measure of cholesterinemia can be used in determining accurately the prognosis.

CAsE 12.-Male, aged 23; no fever; no jaundice; considerable edema; blood pressure, 194; eyegrounds, normal; urine shows many hyaline and granular casts, and a heavy trace of albumin: Wassermann, negative; no evidence of impending uremia. The blood, which was turbid and chylous, showed $7.97 \mathrm{mg}$. of cholesterol, the highest figure I have ever obtained in a case of nephritis. At the same time, the blood showed nonprotein nitrogen, $77.0 \mathrm{mg}$; urea nitrogen, 24; uric acid, 2.3; creatinin, 5.3; sugar, 0.124 per cent. Two weeks later, with conditions practically the same, except for some languor, the following figures were found: Cholesterol, $5.02 \mathrm{mg}$; nonprotein nitrogen, 106; urea nitrogen, 55 ; uric acid, 7 ; creatinin, 13.6 ; sugar, 0.172 per cent.; carbon dioxid combining power, 18 volumes per hundred c.c.; blood indican, ++ , and only a trace of phenolsulphonephthalein elimination. These figures distinctly show that retention of waste products is occurring.

Four days later, the patient had three convulsions and was stuporous, the 1)lood figures were as follows: Cholesterol, $6.40 \mathrm{mg}$; nonprotein nitrogen, 111 ; urea nitrogen, 67; uric acid, 7 ;. creatinin, 14.1; sugar, 0.144 per cent.; carbon dioxid combining power 14 volumes; indican, ++++ . The following day, the patient's general condition was much improved (note the last rise in the cholesterinemia) and remained so for about ten days, but during the interval the following figures were obtained: Cholesterol, $4.97 \mathrm{mg}$. (a considerable fall); nonprotein nitrogen, 112; urea nitrogen, 63 ; uric acid, 5.5; creatinin, 11.1 ; sugar, 0.18 per cent.; indican, ++++ , and no elimination of phenolsulphonephthalein.

At this stage of his illness, the patient again became worse, convulsions of increasing severity and duration, with dulness and apathy set in, and the following figures were obtained: Cholesterol, $3.39 \mathrm{mg}$; nonprotein nitrogen, 125 ; urea nitrogen, 59 ; uric acid, 12.5 ; creatinin, 11.6 ; sugar, 0.120 per cent.; carbon dioxid combining power, 24 volumes; indican, ++++ . Five days later, with convulsions, stupor and restlessness continuing, the following figures were obtained: Cholesterol, $3.38 \mathrm{mg}$; nonprotein nitrogen, 117; urea nitrogen, 72 ; uric acid, 4.2 ; creatinin, 12.3; sugar, 0.153 per cent.; carbon dioxid combining power, 29.6 volumes (treatment directed to combat the acidosis); indican, ++++ . The following day convulsions became very frequent, stupor deepened into coma, and the patient died. Blood obtained hefore death gave the following figures: Cholesterol, $2.49 \mathrm{mg}$; nonprotein nitrogen, 144 ; urea nitrogen, 100 ; uric acid, 12.5 ; creatinin, 13.4 ; sugar, 0.20 per cent.; carbon dioxid combining power, 24 volumes; indican, ++++ .

Here, then, is a case in which the cholesterinemia fell gradually from 7.97 to $2.49 \mathrm{mg}$. in the course of six weeks, while the nonprotein nitrogen constituents of the blood steadily increased in amount. It is the fall in the measure of cholesterinemia that is the significant feature in estimating the prognosis in any given case.

It has long been my belief that the lipoids of the blood play a rôle analogous to an antitoxin, and are intimately associated in immunologic processes. The lipoids seem to act as a protection to the animal organism, and are known to counteract certain poisonous substances. Recent literature abounds in substantiation of this belief. 


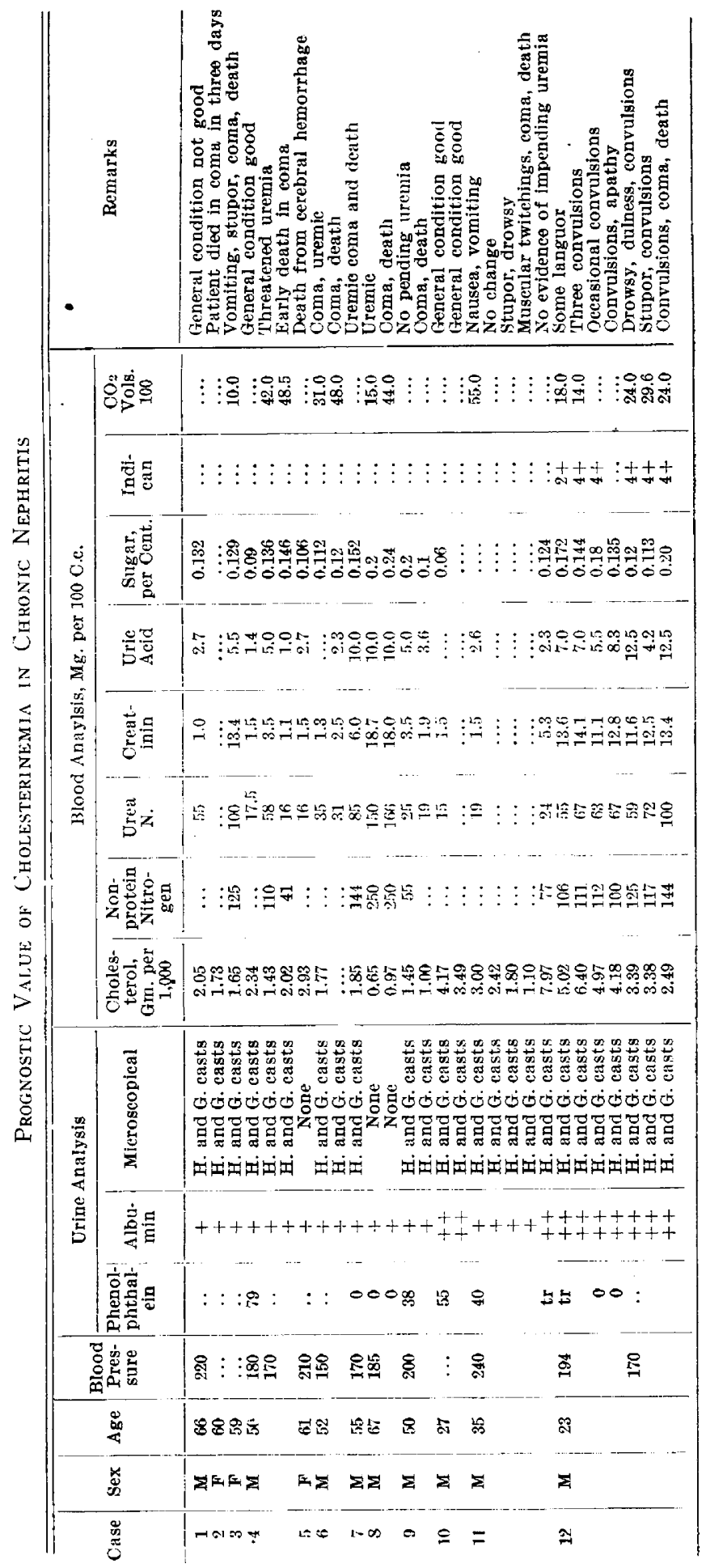


"Cholesterol is no fortuitous component of the organism." 2 Barbary ${ }^{3}$ believes that cholesterol stimulates the production of antibodies. In his hospital at Nice, where during twenty months he cared for 767 wounded and 797 sick soldiers, he had a strikingly low mortality: four deaths among the wounded and nine deaths among the sick. He attributes the good results obtained to the general use of the following mixture: Cholesterin, $0.2 \mathrm{gm}$; camphor, $0.5 \mathrm{gm}$; strychnin sulphate, $0.0005 \mathrm{gm}$. ; in 5.0 c.c. of olive oil washed in alcohol.

Urra $^{4}$ found that cholesterol neutralizes toxins. He found that a hypercholesterinemia is to be found in all severe toxic processes at the time the toxemia is being overcome, referring especially to nephritis and typhoid fever. Several years ago" I showed that a definite hypocholesterinemia exists during the fever period of typhoid, a slow return to normal as the fever approaches normal, and a decided hypercholesterinemia during convalescence.

Manfredi ${ }^{6}$ has shown that the addition of cholesterol to cultures of typhoid bacilli (even in amounts less than are found in the blood stream) checked the growth of the organism. Other organisms, such as the colon bacillus, the streptococcus of erysipelas and puerperal fever, the staphylococcus, diphtheria and cholera bacilli, were also checked in their growth. The hypercholesterinemia of pregnancy is acknowledged to be a fact, and as the result of the study of more than one thousand cases, I believe a relative hypercholesterinemia is to be found in puerperal fever.

Goormaghtigh ${ }^{7}$ has shown that the suprarenal supplies cholesterol to the blood stream in infectious diseases, and in grave infections, terminating fatally, the reserve of cholesterol was demonstrated to be exhausted.

Morato and Villanueva ${ }^{8}$ have shown that the injection of cholesterol with typhoid vaccine hastened the production of antibodies and agglutinins. They cite researches of others who have shown the benefit following the administration of cholesterol in those conditions in which a hypocholesterinemia is found.

Cholesterol has been shown to be antitoxic to the venom of a viper and a cobra. It has been shown to neutralize the toxin of sausage

2. Editorial, J. A. M. A. 62:620 (Feb. 21) 1914.

3. Barbary: Cholesterin and Camphor to Mobilize and Reinforce the Natural Defensive Powers, Bull. de l'Acad. de méd., Par. 76:221.

4. Urra: The Defensive Lipoids, Semana méd. 24:506.

5. Hennes: Cholesterinemia. Proc. New York Pathol. Soc. 13: No. 7.

6. Manfredi: Effect of Cholesterol in Culture Medium on Development of Germs, Riforma méd., Naples 33:849.

7. Goormaghtigh: The Functioning of the Suprarenal Capsules in Infections, Arch. méd., Belges 70:697.

8. Morato and Villanueva: Cholesterol a Factor in Immunity and in the Production of Agglutinins, Med. Ibera, Madrid 9: No. 107. 
poisoning and to attenuate tuberculin, and it has been used in the treatment of tuberculosis and various anemias.

These references to the literature have been presented in justification of my belief that cholesterol plays some protective rôle in the animal organism. The prognostic value of the measure of cholesterinemia in chronic nephritis becomes of greater interest and importance because of these facts. The researches of the past years seem to justify a conclusion I came to eight years ago: "Cholesterinemia seems to be of prognostic importance in some diseases and an indicator of their severity. Researches in cholesterinemia are still in their infancy. - Much work is necessary before the many problems this lipoid presents in its relation to the organism, both under normal and pathologic conditions, can be solved. We have yet to determine more exactly, above all, its origin and destiny, the physiology and pathology of the quantitative variations in the blood, the significance of these variations and the possible rôle cholesterin can play in therapy." ' Many of these problems have since been solved.

I wish to acknowledge my indebtedness to Mr. A. Bernhard, in charge of the chemical laboratory of the Lenox Hill Hospital, for the chemical examinations of the blood in the cases reported.

445 Milwankee Street. 